\title{
Obtaining Highly Crystalline Barium Sulphate Nanoparticles via Chemical Precipitation and Quenching in Absence of Polymer Stabilizers
}

\author{
Ángela B. Sifontes, ${ }^{1}$ Edgar Cañizales, ${ }^{2}$ Jhoan Toro-Mendoza, ${ }^{3}$ \\ Edward Ávila, ${ }^{1}$ Petra Hernández, ${ }^{1}$ Blas A. Delgado, ${ }^{2}$ G. Brenda Gutiérrez, ${ }^{1}$ \\ Yraida Díaz, ${ }^{3}$ and Eliandreina Cruz-Barrios ${ }^{3}$ \\ ${ }^{1}$ Centro de Química, Instituto Venezolano de Investigaciones Científicas (IVIC), Apartado 20632, Caracas 1020-A, Venezuela \\ ${ }^{2}$ Área de Análisis Químico Inorgánico, PDVSA, INTEVEP, Los Teques 1070-A, Venezuela \\ ${ }^{3}$ Centro de Estudios Interdisciplinarios de la Física, Instituto Venezolano de Investigaciones Científicas (IVIC), Apartado 20632, \\ Caracas 1020-A, Venezuela
}

Correspondence should be addressed to Ángela B. Sifontes; angelasifontes@gmail.com

Received 26 November 2014; Revised 9 February 2015; Accepted 10 February 2015

Academic Editor: Yong Zhu

Copyright (C) 2015 Ángela B. Sifontes et al. This is an open access article distributed under the Creative Commons Attribution License, which permits unrestricted use, distribution, and reproduction in any medium, provided the original work is properly cited.

\begin{abstract}
Here we report the synthesis of barium sulphate $\left(\mathrm{BaSO}_{4}\right)$ nanoparticles from $\mathrm{Ba}(\mathrm{OH})_{2} / \mathrm{BaCl}_{2}$ solutions by a combined method of precipitation and quenching in absence of polymer stabilizers. Transmission electron microscopy (HRTEM), Fourier transforms infrared spectroscopy (FTIR), and X-ray diffraction (XRD) were employed to characterize the particles. The Scherrer formula was applied to estimate the particle size using the width of the diffraction peaks. The obtained results indicate that the synthesized material is mainly composed of nanocrystalline barite, with nearly spherical morphology, and diameters ranging from 4 to $92 \mathrm{~nm}$. The lattice images of nanoparticles were clearly observed by HRTEM, indicating a high degree of crystallinity and phase purity. In addition, agglomerates with diameters between 20 and $300 \mathrm{~nm}$ were observed in both lattice images and dynamic light scattering measurements. The latter allowed obtaining the particle size distribution, the evolution of the aggregate size in time of $\mathrm{BaSO}_{4}$ in aqueous solutions, and the sedimentation rate of these solutions from turbidimetry measurements. A short discussion on the possible medical applications is presented.
\end{abstract}

\section{Introduction}

The baryte group consists of baryte, celestine, anglesite, and anhydrite. Baryte is a sulfate of barium with chemical formula $\mathrm{BaSO}_{4}$. It is generally white or colorless, chemically inert, insoluble in water, with high density, and the main source of barium. Although baryte contains a "heavy" metal (barium), it is not considered to be a toxic chemical reagent by most governments because of its extreme insolubility [1]. Commonly, baryte is used for the production of barium hydroxide for sugar refining and as a white pigment for textiles, paper, and paint [2]. It is also suitable for other purposes due to its high specific gravity (4.5), opaqueness to X-rays, inertness, and whiteness [3]. Recently, more attention has been paid on the massive production of $\mathrm{BaSO}_{4}$ nanoparticles for its multiple applications in the oil industry, electronics, TV screen, glass, car filters, paint industry, and ceramics [4] as well as in medical applications. In particular, barium sulfate suspension in water is used as the universal contrast medium for the examination of the upper gastrointestinal tract [5]. Currently, the development of nanoparticle-based X-ray contrast agents is providing an increasing contribution to the field of diagnostic and molecular imaging. For example, the nanoparticles surface can be modified to enhance their specificity by attaching targeting moieties, increase their circulation half-life by adding appropriate coatings (e.g., polymers, silica), and 
improve their functionality by adding other components, including fluorescent markers and therapeutic agents [6]. However, although significant progress has been made in preparing stable nanoparticles, their industrial production can present serious difficulties that limit their potential applications. For example, $\mathrm{BaSO}_{4}$ nanoparticles tend to form aggregates due to the high ratio surface area-volume. Expensive costs and complex methodologies also attempt against massive industrial production [3].

Among the methods to obtain nanoscaled materials, chemical synthesis has numerous advantages such as simple technique, low costs, less instrumentation, doping, and high yield [4]. These methods could be classified as (a) direct precipitation [7], (b) microemulsion [8], (c) membrane separation [9], and (d) organic modification [10]. In general, particle sizes between 16 and $50 \mathrm{~nm}$ can be obtained. Nonetheless, precipitation seems to be the most suitable method due to its simple procedure and ease of mass production, and less apparatus is required [11]. In spite of this, the difficulties in controlling the size, polydispersity, and strong tendency to aggregate could hinder their potential applications [11]. In this sense, it is important to find more efficient methodologies of synthesis. Accordingly, in this paper we report the synthesis and characterization of barium nanoparticles by a chemical precipitation route, in the absence of polymer stabilizers.

\section{Materials and Methods}

2.1. Chemicals and Instruments. The starting reagents barium hydroxide, barium chloride, and sulfuric acid were used as received. Double distilled water was used in all experiments. The prepared barium sulfate was characterized by transmission electron microscopy using a JEOL JEM-2100 microscope with $\mathrm{LaB}_{6}$ filament (accelerating voltage of $200 \mathrm{kV}$ ). The samples were prepared by suspending the powders in an ethanol-based liquid and pipetting the suspension onto a carbon/collodion coating. Fourier transform infrared (FTIR) spectra were measured with a Perkin Elmer 100 spectrometer (in the range of $2000-500 \mathrm{~cm}^{-1}$ ) by incorporating the samples in $\operatorname{KBr}(1: 99 \mathrm{mg})$ disks to confirm the characteristic vibrational bands. X-ray diffraction (XRD) patterns of $\mathrm{BaSO}_{4}$ were recorded on a panalytical diffractometer, model X'Pert Pro, and CuK- $\alpha$ radiation in the $2 \theta$ range between 5 and $80^{\circ}$, operating at $40 \mathrm{kV}$ and $20 \mathrm{~mA}$. Phases were identified using the MATCH! 2 program (version 2.2.3, Build 361) Crystal Impact, coupled to the Release 2011 PDF-2 database [12]. PDF card number 024-1035 (or ICSD card number 33730) was used to carry out the Rietveld refinement to all the peaks [13], which were in accordance with the reported values in the literature. The structure of $\mathrm{BaSO}_{4}$ was refined with the Rietveld (1969) program GSAS [14] using the graphical interface EXPGUI [15]. The peak shapes were modeled using the pseudo-Voigt peak shape function 3, which included the axial divergence correction at low angle [16]. Background was initially determined manually and then modeled using the Chebyshev polynomial function. The isotropic atomic displacement parameters were refined as one overall $U_{\text {iso }}$ for the nonoxygen atoms starting from a value of $0.05 \AA^{2}$.
TABLE 1: Selected peaks employed to calculate the particles size with Scherrer's equation. These are the nine first peaks that appearance powder pattern of $\mathrm{BaSO}_{4}$ synthesized.

\begin{tabular}{lccccc}
\hline$h k l$ & $2 \theta /^{\circ}$ & $d / \AA$ & Int./U.A. & Rel. Int./\% & FWHM/2 $\theta^{\circ}$ \\
\hline 200 & 19.9969 & 4.43666 & 211.47 & 13.68 & 0.0895 \\
011 & 20.4650 & 4.33622 & 513.75 & 33.24 & 0.0892 \\
111 & 22.8076 & 3.89586 & 795.40 & 51.46 & 0.0882 \\
210 & 23.5789 & 3.77013 & 155.81 & 10.08 & 0.0879 \\
002 & 24.8785 & 3.57606 & 448.89 & 29.04 & 0.0875 \\
210 & 25.8694 & 3.44128 & 1521.90 & 98.46 & 0.0872 \\
120 & 26.8582 & 3.31679 & 1090.44 & 70.55 & 0.0870 \\
211 & 28.7665 & 3.10096 & 1545.73 & 100.00 & 0.0868 \\
112 & 31.6281 & 2.83364 & 535.66 & 34.65 & 0.0868 \\
\hline
\end{tabular}

Nevertheless, one constraint was applied to $U_{\text {iso }}$ for modeling the isotropic atomic displacement parameters of oxygen atom following riding-model. The mean grain size $(D)$ of the prepared $\mathrm{BaSO}_{4}$ nanoparticles was estimated from X-ray line broadening of the listed reflections in Table 1, using the Scherrer equation [17] $(D=K \lambda /(\beta \cos \theta)$ where $\lambda$ is the wavelength of the X-ray radiation, $K$ is a constant taken as $0.89, \theta$ is the diffraction angle, and $\beta$ (rad) is the full width at half-maximum (FWHM)).

Both initial particle size distribution and particles size variation were characterized by dynamic light scattering measurements (DLS) on a Brookhaven BI 9000AT goniometer. Measurements were carried out at a fixed angle of $90^{\circ}$ using a $633 \mathrm{~nm}$ laser. The sedimentation rate was obtained from Turbiscan LAB software in scanning mode. The light source is an electro luminescent diode in near infrared $(880 \mathrm{~nm})$. Two synchronous optical sensors receive the transmitted light through the sample $\left(180^{\circ}\right)$ and the backscattered light by the sample $\left(45^{\circ}\right)$. The sample was dispersed in a deionized water solution at a phase fraction of $0.1 \% \mathrm{w} / \mathrm{v}$. The suspension was sonicated during $15 \mathrm{~min}$ before measurement.

2.2. Preparation of the Samples. The direct precipitation (DP) procedure followed consists in adding a $\mathrm{Ba}(\mathrm{OH})_{2} \cdot 8 \mathrm{H}_{2} \mathrm{O}$ solution to $\mathrm{BaCl}_{2} \cdot 2 \mathrm{H}_{2} \mathrm{O}$ in presence of water. The molar ratio of $\mathrm{Ba}(\mathrm{OH})_{2}$ to $\mathrm{BaCl}_{2}$ was $3: 1$. The resulting solution was stirred at room temperature while a sulfuric acid solution $(50 \%(\mathrm{v} / \mathrm{v}))$ was added (step 1 , precipitation). The precipitated solid material was washed and filtered four times with hot distilled water (step 2). The sample was frozen at $-26^{\circ} \mathrm{C}$ for 24 hours. Subsequently, the supernatant liquid was carefully decanted and then a volume of distilled water was added again. This procedure was repeated 5 times. The final step involved a freeze-dry cycle and was oven-dried at $105^{\circ} \mathrm{C}$ for 12 hours (step 3, purification) [18]. All the experiments were performed three times in order to observe reproducibility.

\section{Results and Discussion}

Figure 1 shows the XRD pattern and final Rietveld plot of the synthesized sample. It is shown that the solid is composed of baryte. The $\mathrm{BaSO}_{4}$ crystalized in orthorhombic crystals, space 


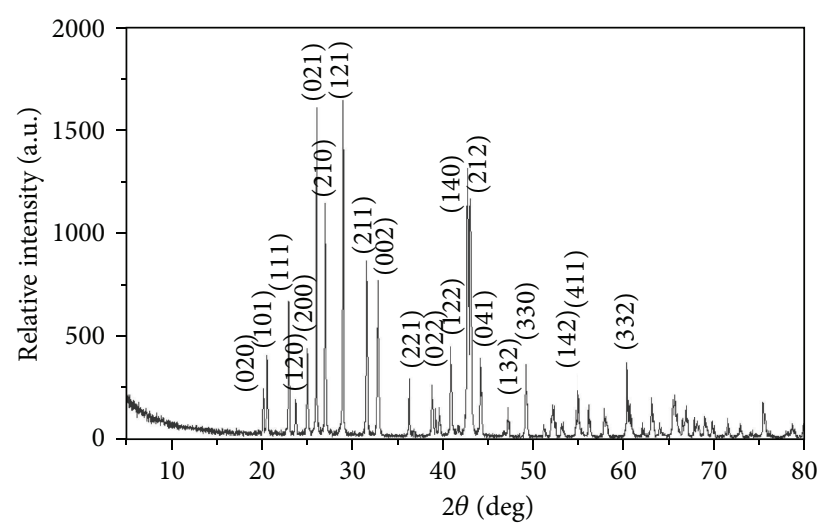

(a)

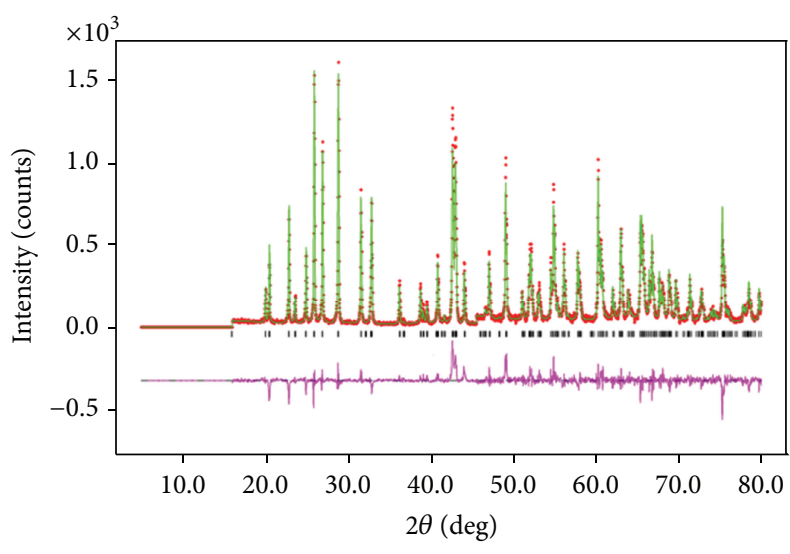

(b)

FIGURE 1: XRD pattern of synthesized sample (a) and final Rietveld plot for $\mathrm{BaSO}_{4}$ (b). The continuous green line represents the calculated pattern and the red discontinuous line is the observed pattern.

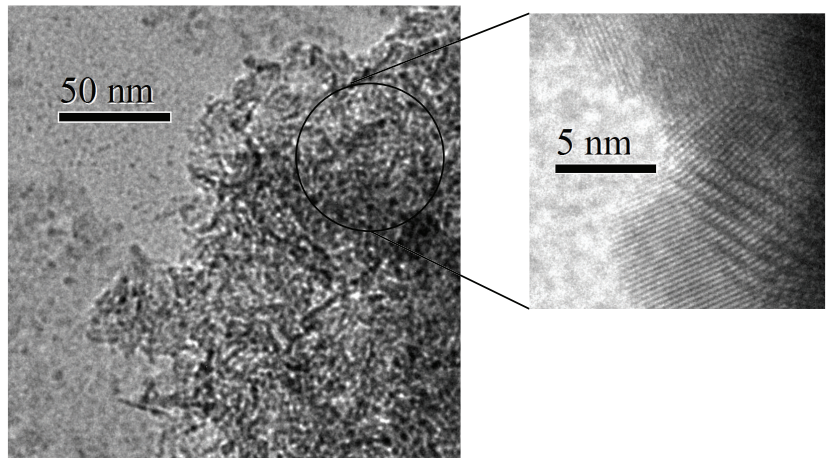

(a)

(b)

FIGURE 2: TEM image of $\mathrm{BaSO}_{4}$ nanoparticles (a); HRTEM image showing lattice fringes of $\mathrm{BaSO}_{4}$ nanoparticles (b).

group Pnma, with cell parameters $a=8.875 \AA, b=5.453 \AA$, and $c=7.154 \AA$. The final Rietveld refinement with the reported model gave figures of merit: $R_{p}=0.1135, R_{\mathrm{wp}}=$ $0.1433, R_{\exp }=0.014, R_{F}{ }^{2}=0.0945$ (249 reflections), and $\chi^{2}=$ 1.41. Finally, the Scherrer equation was used to estimate the particle size by averaging the value of FWHM for the first nine lines of the powder pattern of $\mathrm{BaSO}_{4}$ (see Table 1). Thus, the average particle size estimated for the $\mathrm{BaSO}_{4}$ synthesized in this work was $91.72 \mathrm{~nm}$.

TEM and HRTEM images (Figures 2-6) show that the sample was composed of both isolated and aggregated particles. The morphology of $\mathrm{BaSO}_{4}$ particles is nearly spherical. The nanoparticles size determined from micrographies is between 2.6 and $20 \mathrm{~nm}$, with an average size of $14.5 \mathrm{~nm}$. Besides, large $(>20 \mathrm{~nm})$ fully crystallized structures are observed. Typical sizes of aggregates were found to be about 11-300 nm (Figure 3).

In Figure 4 HRTEM analysis shows that the lattice fringes with an in interlayer distance of $0.327 \mathrm{~nm}$ is close to the $0.331 \mathrm{~nm}$ lattice spacing of the $\{210\}$ crystal planes of barite phase. This was confirmed by fast Fourier transformation (FFT) patterns, which is also in accordance with XRD results. For this study, noise reduction was performed on the squarelabeled area of Figure 4(a). Masking was applied on the intense spots of the FFT power spectrum. The resulting image after performing inverse FFT on the masked power spectrum is showed in Figure 4(d).

In Figure 5(a) (nanoparticles 1,2) HRTEM analysis shows that the lattice fringes with an interlayer distance of $0.338 \mathrm{~nm}$ correspond to lattice spacing of the (210) crystal planes of $\mathrm{BaSO}_{4}$, which is in accordance with XRD results. Figures 6(b) and 6(c) show a FFT of Figure 5(a), and the diffraction points can be indexed to the (210) plane. Masking was also applied on Figures 5(b) and 5(c), specifically on the intense spots of the FFT power spectrum. The resulting image (Figures 5(d) and 5(e)) was obtained after performing inverse FFT on the masked power spectrum (Figures 5(f) and 5(g)).

In the HRTEM image (Figure 6), the planar spacing obtained is $0.355 \mathrm{~nm}$, corresponding to spacing for (200) planes of orthorhombic structure $\mathrm{BaSO}_{4}$. All the planar 


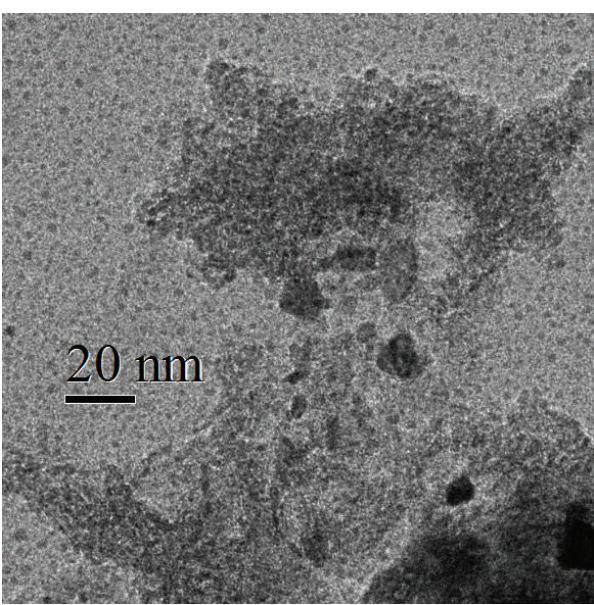

(a)

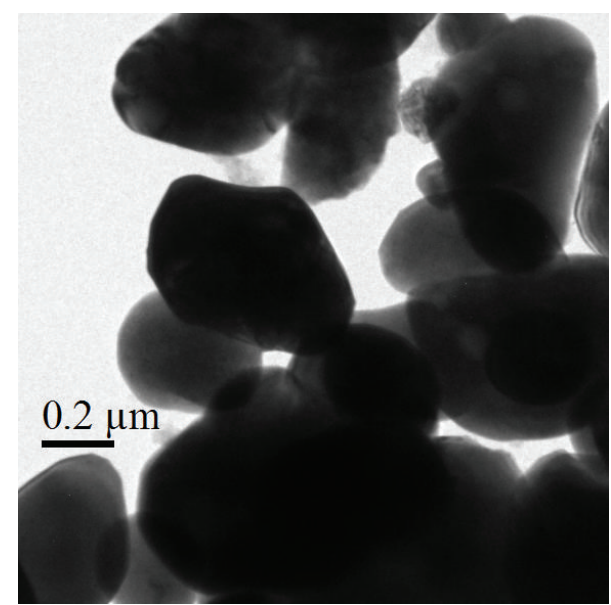

(b)

FIgURE 3: TEM image of $\mathrm{BaSO}_{4}$ nanoparticles (a); typical aggregates (b).

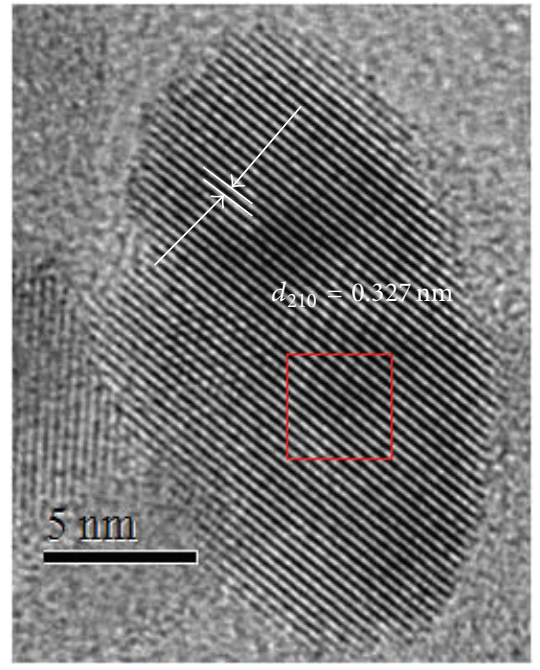

(a)

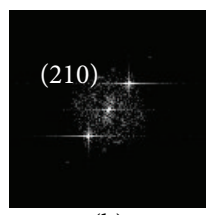

(b)

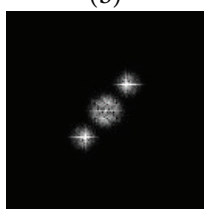

(c)

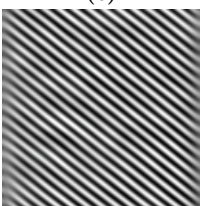

(d)

FiguRE 4: HRTEM image of $\mathrm{BaSO}_{4}$ nanoparticle (a); FFT power spectrum (b); masked power spectrum FFT (c); image after performing inverse FFT on the masked power spectrum (d).

spacing determined from HRTEM images are in accordance with the database of Joint Committee on Powder Diffraction Standards (JCPDS) card 024-1035.

Figure 7 shows the FT-IR spectra of the synthesized $\mathrm{BaSO}_{4}$ nanoparticles. It was previously mentioned that the sulfate group has four fundamental vibrational modes attributed to one nondegenerate mode $\left(\nu_{1}\right)$, one doubly degenerate mode $\left(\nu_{2}\right)$, and two triply degenerate modes $\left(\nu_{3}\right.$ and $\left.v_{4}\right)$. In general, the IR spectrum of baryte exhibits several significant bands [19]. Two intense bands correspond to asymmetric stretching and bending $\left(\nu_{3}\right.$ and $\left.v_{4}\right)$, and two weak ones correspond to symmetric stretching and bending $\left(\nu_{1}\right.$ and $\left.v_{2}\right)$ [19]. In the FT-IR spectra the bands centered at $1073-1185 \mathrm{~cm}^{-1}$ and the shoulder at $982 \mathrm{~cm}^{-1}$ were assigned to symmetric stretching vibration of $\mathrm{SO}_{4}{ }^{2-}$ group. At $1636 \mathrm{~cm}^{-1}$ the stretching vibration $v_{3}$ of $\mathrm{SO}_{4}{ }^{2-}$ group was identified. The peaks obtained at 609 and $637 \mathrm{~cm}^{-1}$ were attributed to the out-of-plane bending vibration of the $\mathrm{SO}_{4}{ }^{2-}[10$, 19]. The absorption peaks appeared at $3420 \mathrm{~cm}^{-1}$ are due to antisymmetric stretching vibrations of $\mathrm{H}_{2} \mathrm{O}$ molecules localized on vacant sites of $\mathrm{Ba}$. The peaks around $2000 \mathrm{~cm}^{-1}$ are overtones and a combination of stretching and bending vibrations bands of the sulphur-oxygen [20].

3.1. Particle Size Distribution, Aggregates Size Evolution, and Sedimentation Rate. Figure 8 shows the particle size distribution from dynamic light scattering (DLS) measures of an aqueous suspension of $\mathrm{BaSO}_{4}$ nanoparticles. As can be observed, the main peak appears around $800 \mathrm{~nm}$ with an average size of $2.23 \pm 0.72 \mu \mathrm{m}$. Even though the micrographies 


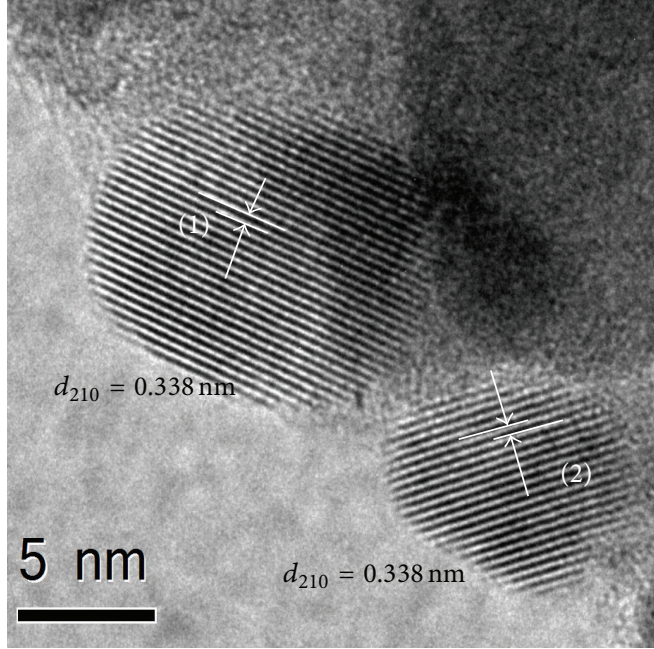

(a)

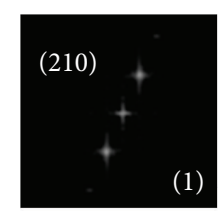

(b)

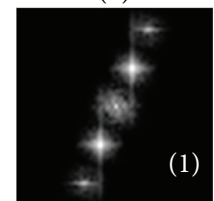

(d)

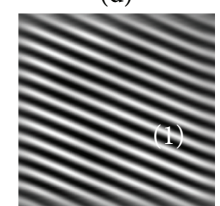

(f)

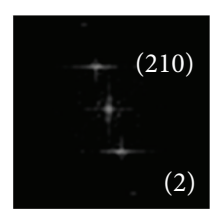

(c)

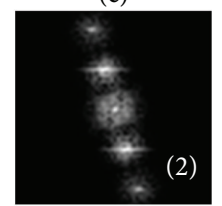

(e)

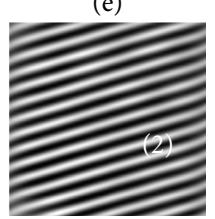

(g)

Figure 5: HRTEM image of $\mathrm{BaSO}_{4}$ nanoparticles (a); FFT image (b) (particle 1), (c) (particle 2); masked power spectrum FFT (c) (particle 1), (d) (particle 2); image after performing inverse FFT on the masked power spectrum (c) and (d).

indicate a particle size less than $100 \mathrm{~nm}$, the distribution shows particles sizes above 5 microns, an indicative that DLS yields an average radius of aggregates formed by several particles. The strong tendency to form aggregates was corroborated and the results are shown in Figure 9, where the time evolution of both radius and percentage of polydispersity is plotted. The average size evolves linearly with time from $1200 \mu \mathrm{m}$ to $4200 \mu \mathrm{m}$ in $1 \mathrm{~h}$. However, the percentage of polydispersity fluctuates around $30 \%$.

Due to the large size of aggregates, it is expected to observe sedimentation and the results from turbidimetry can be observed in Figure 10. The photon mean free path (l) represents the mean distance travelled by photons before undergoing a diffusion phenomenon. Thus, the measurement is performed by the Turbiscan by sending a light beam though the cell and detecting the photons crossing the dispersion, without being diffused. Therefore as more photons cross the cell, more important is the $l$ value and the sample is more translucent, an indicative that less particles are in the beam path. The initial slope of Figure 10, corresponding to the sedimentation rate, was $412 \mu \mathrm{m} / \mathrm{min}$, with a correlation coefficient of 0.99 . The total average sedimentation rate in a $60 \mathrm{~min}$ measurement was $171 \mu \mathrm{m} / \mathrm{min}$.

The combined effect of forming aggregates and sedimentation could limit possible medical applications of $\mathrm{BaSO}_{4}$ nanoparticles. Nevertheless, this study was processed without any additives (including stabilizers, antioxidants, and lubricants). In this sense, in general, barium sulfate suspension oral for gastric and colonic radiological work, containing a physiologically inert stabilizer, consists of a solution of a water-soluble cellulose ether and carboxymethylcellulose and also contains a suitable penetrant of cationic origin as lauryl sulfoacetate [21]. Besides, in order to improve the possibilities of application, further studies in particle-particle interactions and the main mechanisms responsible for coarsening are necessary to implement new methodologies to obtain more kinetically stable systems. In this sense, our procedure could be optimized in order to low the polydispersity index around a particle size below $10 \mathrm{~nm}$. It is also noticeable that the starting reagents have low impact toxicity, compared to others which, in some cases, make use of benzene [22]. This latter is an additional advantage of our procedure in order to use the synthesized nanoparticles in medical applications.

3.2. Formation Mechanism of $\mathrm{BaSO}_{4}$ Nanoparticles. The synthesis of barium sulfate was carried out using a mix of $\mathrm{Ba}(\mathrm{OH})_{2} / \mathrm{BaCl}_{2}$, where the barium chloride solution was added to increase the concentration of $\mathrm{Ba}^{2+}$ ions (in excess). Subsequently, with the addition of $\mathrm{SO}_{4}{ }^{2-}\left(\mathrm{H}_{2} \mathrm{SO}_{4}\right), \mathrm{BaSO}_{4}$ crystals were gradually formed.

The increase in $\mathrm{pH}$ could result in an increase in the negative charge of $\mathrm{BaSO}_{4}$. According to Zhang et al., this could be attributed to the adsorption of $\mathrm{OH}^{-}$ions on the positive charge centers of $\mathrm{BaSO}_{4}$ particles [23]. The reaction of barium $\left(\mathrm{Ba}^{2+}\right)$ and sulphate $\left(\mathrm{SO}_{4}{ }^{2-}\right)$ ions into barium sulphate $\left(\mathrm{BaSO}_{4}\right)$ is $[20,24,25]$

$$
\mathrm{Ba}^{2+}(\mathrm{aq})+\mathrm{SO}_{4}{ }^{2-}(\mathrm{aq}) \longrightarrow \mathrm{BaSO}_{4}(\mathrm{~s}) \downarrow
$$

In general, a precipitation process is described by processes that include the creation of a supersaturation level followed by the generation of nuclei (nucleation) and the subsequent growth [8]. A number of secondary processes like aging, ripening, agglomeration, and breakage might occur depending on the nature of the process itself and the chemical compound [8]. Moreover, in a highly supersaturated solution the instability of the system will result in a fast local concentration fluctuation [26]. Basically, there are three variables that govern the rate of nucleation: the temperature $T$, the degree of supersaturation $S$, and the surface energy $\sigma$. In this sense, if the supersaturation ratio is increased and the surface energy is decreased, the result is a higher nucleation rate, which finally leads to the formation of particles of smaller size $[27,28]$. 


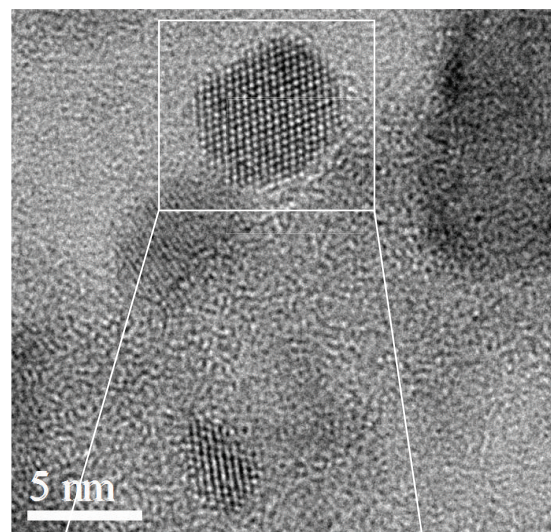

(a)

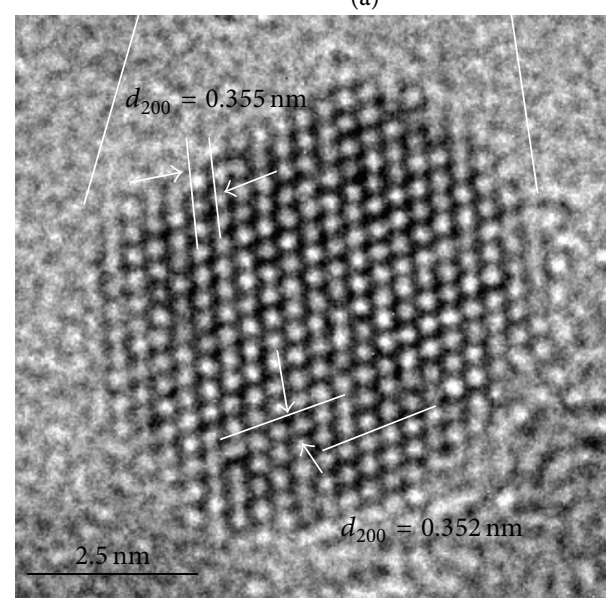

(b)

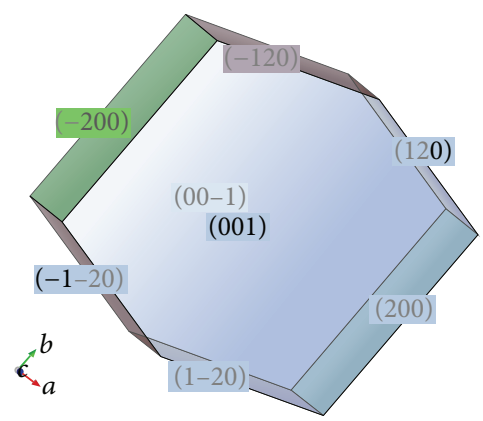

(f)

FIGURE 6: HRTEM image of $\mathrm{BaSO}_{4}$ nanoparticle (a); HRTEM image of $\mathrm{BaSO}_{4}$ nanoparticle (b); a magnification of the section marked with a white square in (a); FFT image (c); masked power spectrum FFT (d); image after performing inverse FFT on the masked power spectrum (e). A crystallographic representation of $\mathrm{BaSO}_{4}$ crystal (f).

The synthesis process involves as second step a quenching. Quenching is simply cooling rapidly to a lower temperature, $-26^{\circ} \mathrm{C}$. The final step in the precipitation process was an artificial aging at $-26^{\circ} \mathrm{C}$ (aging above room temperature). Temperature has a significant influence on solubility and crystal growth of barium sulphate [29]. It is then expected that the growth of particle size is stopped by quenching with cold water.

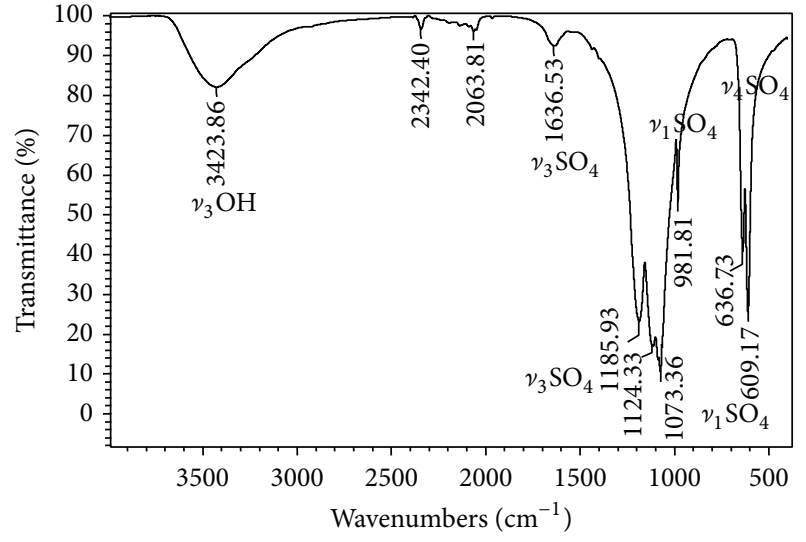

FIGURE 7: FT-IR spectra of $\mathrm{BaSO}_{4}$ nanoparticles.

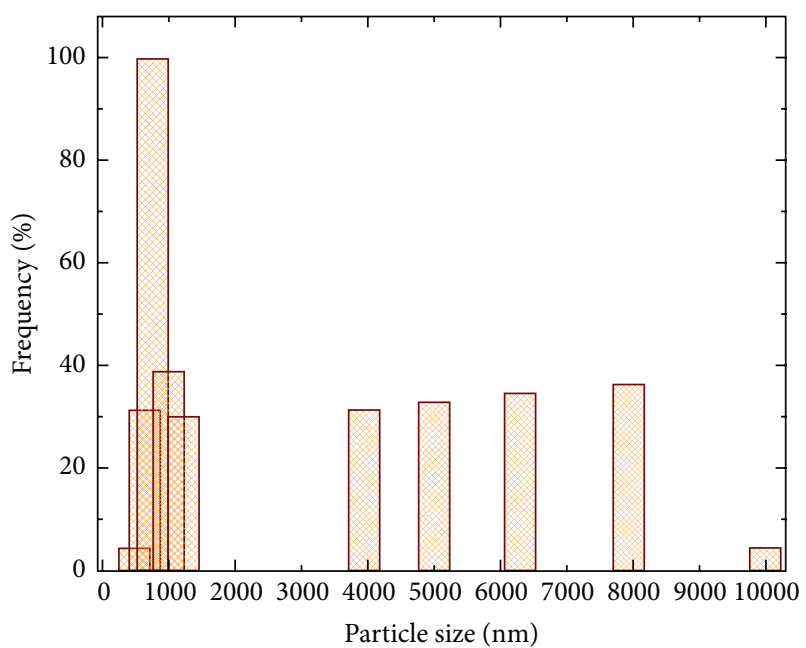

Figure 8: Particle size distribution of $\mathrm{a} \mathrm{BaSO}_{4} / \mathrm{W}$ suspension $(0.1 \%$ $\mathrm{w} / \mathrm{v}$ ) determined by dynamic light scattering.

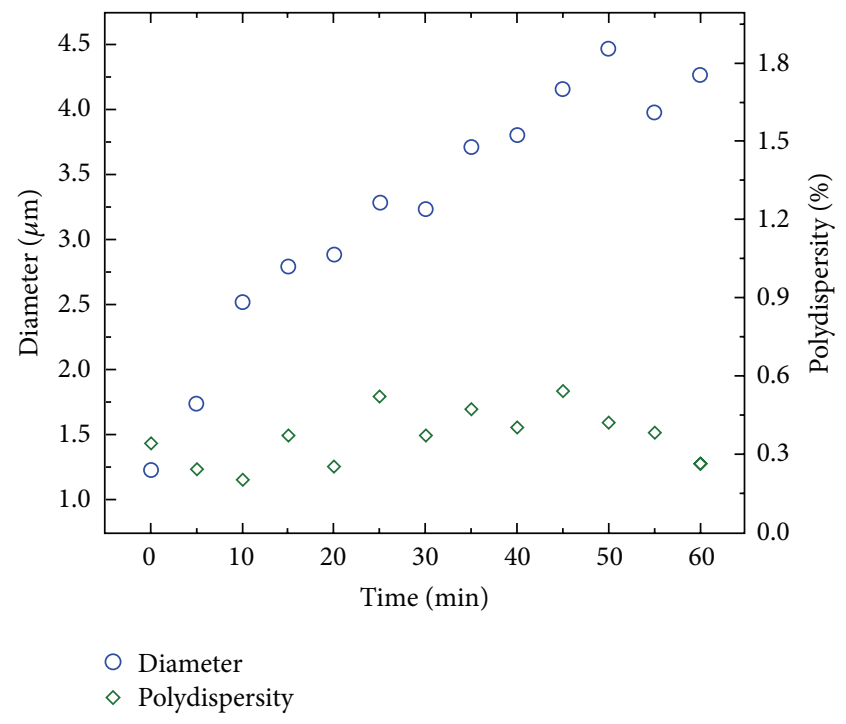

Figure 9: Particle size variation of a $\mathrm{BaSO}_{4} / \mathrm{W}$ suspension $(0.1 \% \mathrm{w} / \mathrm{v})$ determined by dynamic light scattering. 


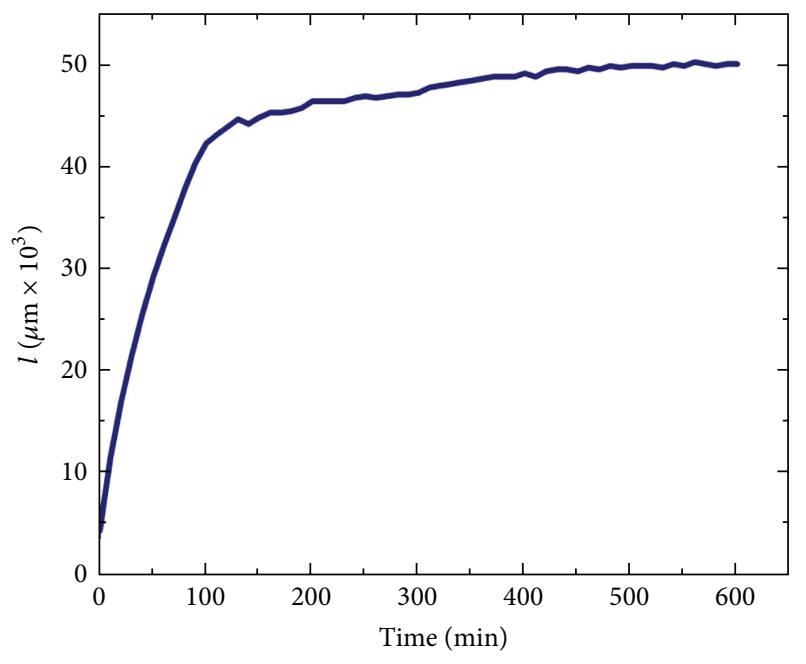

FIGURE 10: Sedimentation rate obtained from the initial slope of the photon mean free path $(l)$ as a function of time.

The results presented here prove that barium sulfate nanoparticles can be prepared in the absence of polymers stabilizers polymeric employing a simple methodology. Moreover, the nanomaterial synthesized has applications as a potential contrast agent for X-ray examination.

\section{Conclusions}

Barium sulphate nanoparticles were synthesized using a precipitation method in absence of polymer stabilizers or solvents. The XRD study shows that the synthesized nanoparticles crystallize in orthorhombic system with space group Pnma. This method allowed obtaining high purity, spheri$\mathrm{cal}$, and morphologically homogeneous nanoparticles. X-ray diffraction, transmission electron microscope (TEM), and high resolution transmission electron microscope (HRTEM) measurements confirmed an average particle size in the range from 4 to $92 \mathrm{~nm}$ and agglomerates with diameters between 20 and $300 \mathrm{~nm}$. FTIR study shows the presence of the sulphate group in the synthesized nanoparticles. Dynamic light scattering and turbidimetry measurement indicates a strong tendency of nanoparticles to form aggregates and precipitate in aqueous solution. Finally, this methodology can be employed to synthesize $\mathrm{BaSO}_{4}$ nanoparticles for possible applications as a potential contrast agent for X-ray examination.

\section{Conflict of Interests}

The authors declare that there is no conflict of interests regarding the publication of this paper.

\section{Acknowledgment}

The authors acknowledge financial support by IVIC through project 1077 and technical assistance of Lic. Liz Cubillán (FTIR).

\section{References}

[1] J. S. Hanor, "Barite-celestine geochemistry and environments of formation," Reviews in Mineralogy and Geochemistry, vol. 40, pp. 193-275, 2000.

[2] J. Dana and W. Ford, Dana's Manual of Mineralogy For the Student of Elementary Mineralogy, the Mining Engineer, the Geologist, the Prospector, the Collector, etc, John Wiley \& Sons, New York, NY, USA, 14th edition, 1929.

[3] Y. Shen, C. Li, X. Zhu, A. Xie, L. Qiu, and J. Zhu, "Study on the preparation and formation mechanism of barium sulphate nanoparticles modified by different organic acids," Journal of Chemical Sciences, vol. 119, no. 4, pp. 319-324, 2007.

[4] V. Ramaswamy, R. M. Vimalathithan, and V. Ponnusamy, "Preparation of barium sulphate nanocrystals in ethanolwater mixed solvents," Journal of Ceramic Processing Research, vol. 12, no. 2, pp. 173-175, 2011.

[5] D. Sutton, A Textbook of Radiology and Imaging, Churchill Livingstone, London, UK, 1980.

[6] J. C. de la Vega and U. O. Häfeli, "Utilization of nanoparticles as X-ray contrast agents for diagnostic imaging applications," Contrast Media \& Molecular Imaging, 2014.

[7] M. Ismaiel and I. Mostafa, "Synthesis of $\mathrm{BaSo}_{4}$ nanoparticles by precipitation method using polycarboxylate as a modifier," American Journal of Nanotechnology, vol. 2, pp. 106-111, 2011.

[8] D. Adityawarman, A. Voigt, P. Veit, and K. Sundmacher, "Precipitation of $\mathrm{BaSO}_{4}$ nanoparticles in a non-ionic microemulsion: identification of suitable control parameters," Chemical Engineering Science, vol. 60, no. 12, pp. 3373-3381, 2005.

[9] G. Wu, H. Zhou, and S. Zhu, "Precipitation of barium sulfate nanoparticles via impinging streams," Materials Letters, vol. 61, no. 1, pp. 168-170, 2007.

[10] A. Gupta, P. Singh, and C. Shivakumara, "Synthesis of $\mathrm{BaSO}_{4}$ nanoparticles by precipitation method using sodium hexa metaphosphate as a stabilizer," Solid State Communications, vol. 150, no. 9-10, pp. 386-388, 2010.

[11] M. I. Nieto, C. Tallón, and R. Moreno, "Synthesis of gammaalumina nanoparticles by freeze drying," Advances in Science and Technology, vol. 45, pp. 223-230, 2006.

[12] H. Putz, MATCH 2-Phase Identification from Powder Diffraction, Version 2.2.3, Crystal Impact, Bonn, Germany, 2014.

[13] H. M. Rietveld, "A profile refinement method for nuclear and magnetic structures," Journal of Applied Crystallography, vol. 2, pp. 65-71, 1969.

[14] A. C. Larson and R. B. von Dreele, GSAS: General Structure Analysis System, Software, The Los Alamos National Laboratory, Los Alamos, NM, USA, 2007.

[15] B. H. Toby, "EXPGUI, a graphical user interface for GSAS," Journal of Applied Crystallography, vol. 34, no. 2, pp. 210-213, 2001.

[16] L. W. Finger, D. E. Cox, and A. P. Jephcoat, "A correction for powder diffraction peak asymmetry due to axial divergence," Journal of Applied Crystallography, vol. 27, no. 6, pp. 892-900, 1994.

[17] S. Phoka, P. Laokul, E. Swatsitang, V. Promarak, S. Seraphin, and S. Maensiri, "Synthesis, structural and optical properties of $\mathrm{CeO}_{2}$ nanoparticles synthesized by a simple polyvinyl pyrrolidone (PVP) solution route," Materials Chemistry and Physics, vol. 115, no. 1, pp. 423-428, 2009.

[18] W. Armarego and D. Perrin, Laboratory Chemical, Butterworth Heinemann, Oxford, UK, 4th edition, 1996. 
[19] B. Prameena, G. Anbalagan, V. Sangeetha, S. Gunasekaran, and G. R. Ramkumaar, "Behaviour of Indian natural baryte mineral," International Journal of ChemTech Research, vol. 5, no. 1, pp. 220-231, 2013.

[20] J. Manam and S. Das, "Thermally stimulated luminescence studies of undoped, $\mathrm{Cu}$ and $\mathrm{Mn}$ doped $\mathrm{BaSO}_{4}$ compounds," Indian Journal of Pure and Applied Physics, vol. 47, no. 6, pp. 435-438, 2009.

[21] R. B. Garland, Patent US3236735 A, 1962.

[22] V. Ramaswamy and R. M. Vimalathithan, "Ponnusamy synthesis of monodispersedbarium sulphate nanoparticles using water-benzene mixed solvent," Advanced Materials Letters, vol. 3, pp. 29-33, 2012.

[23] M. Zhang, B. Zhang, X. Li, Z. Yin, and X. Guo, "Synthesis and surface properties of submicron barium sulfate particles," Applied Surface Science, vol. 258, no. 1, pp. 24-29, 2011.

[24] R. Kieffer, D. Mangin, F. Puel, and C. Charcosset, "Precipitation of barium sulphate in a hollow fiber membrane contactor: part II the influence of process parameters," Chemical Engineering Science, vol. 64, no. 8, pp. 1885-1891, 2009.

[25] A. A. Öncül, K. Sundmacher, A. Seidel-Morgenstern, and D. Thévenin, "Numerical and analytical investigation of barium sulphate crystallization," Chemical Engineering Science, vol. 61, no. 2, pp. 652-664, 2006.

[26] A. Myerson, Handbook of Industrial Crytallization, Butterworth-Heinemann, Boston, Mass, USA, 1993.

[27] N. Tavare, Industrial Crystallization: Process Simulation Analysis and Design, Plenum Press, New York, NY, USA, 1995.

[28] A. Randolph and M. Larson, Theory of Particulate Processes, Academic Press, San Diego, Calif, USA, 2nd edition, 1988.

[29] A. B. B. Merdhah, "Laboratory study on precipitation of barium sulphate in Malaysia," The Open Petroleum Engineering Journal, vol. 2, pp. 1-11, 2009. 

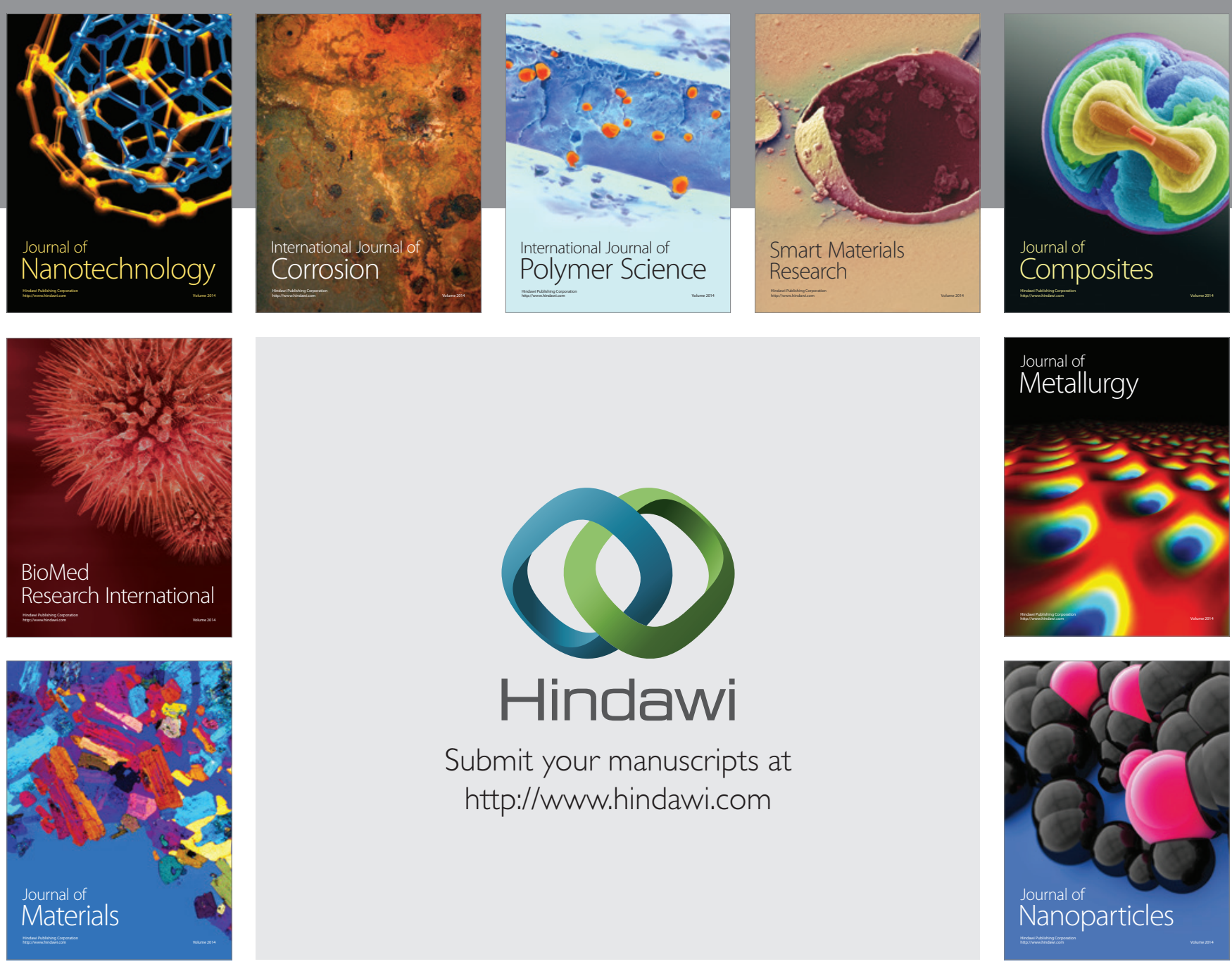

Submit your manuscripts at http://www.hindawi.com
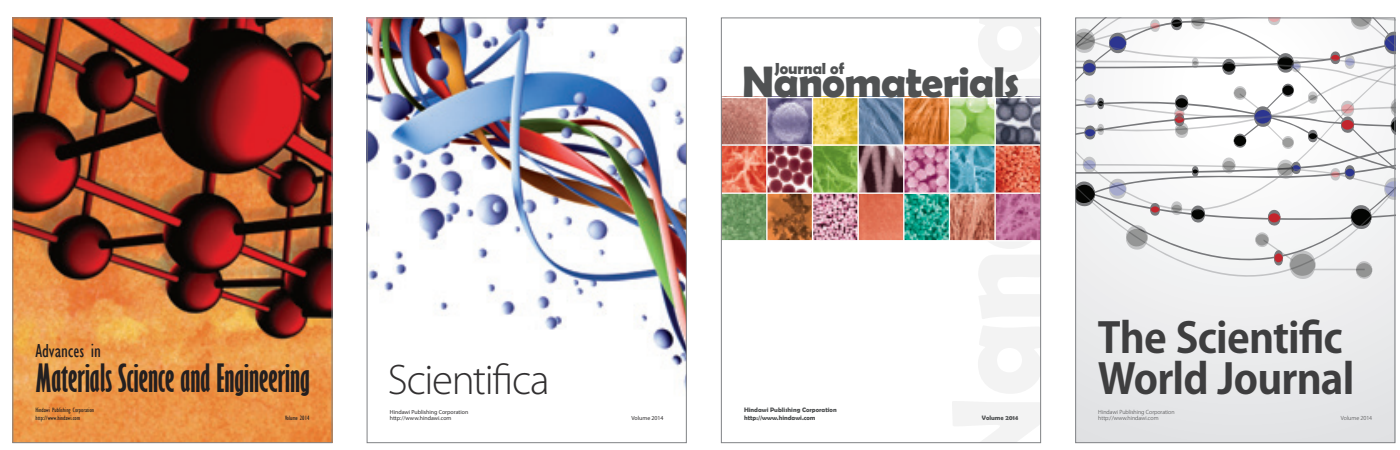

\section{The Scientific World Journal}
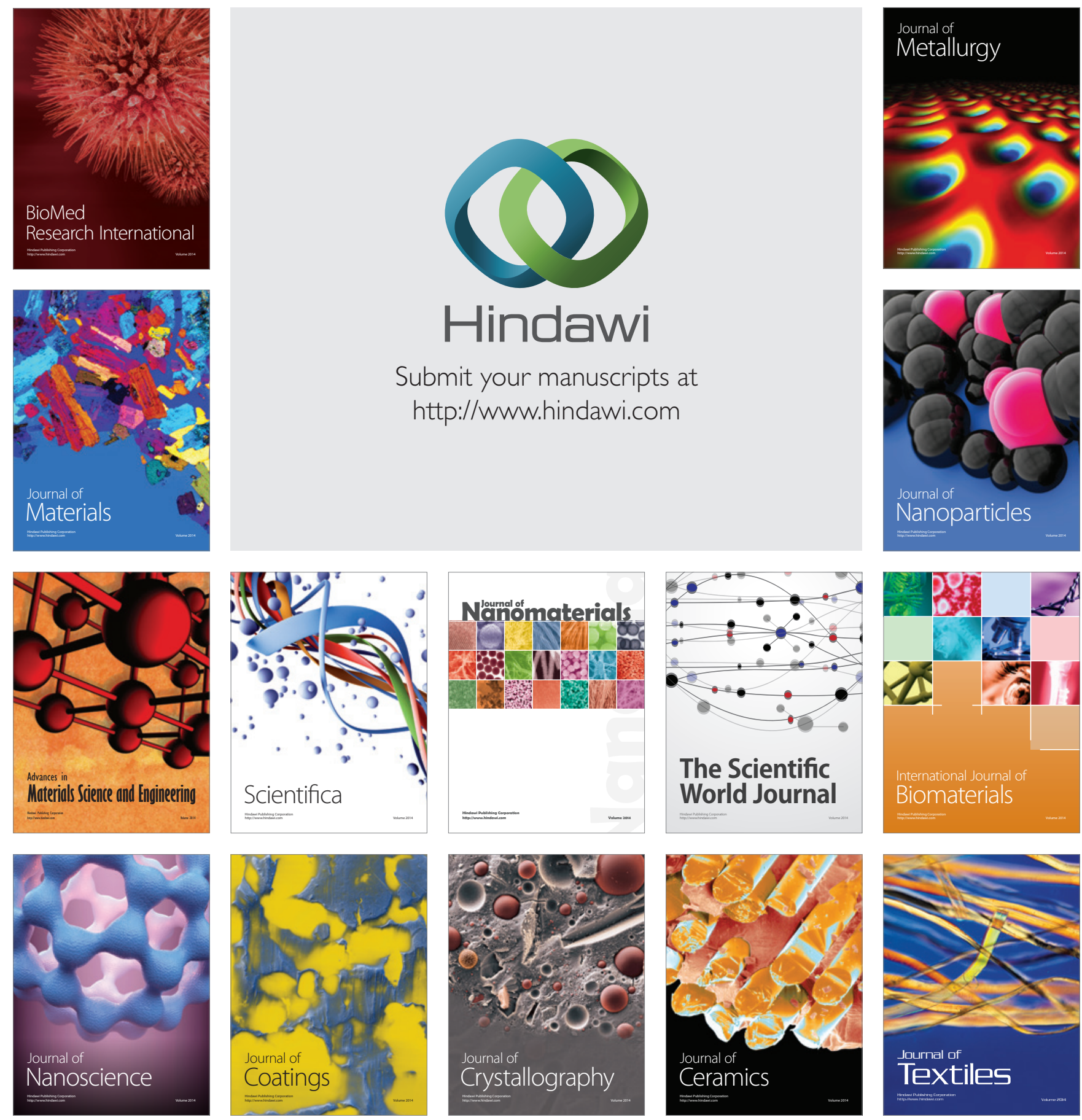